\title{
135 In-vivo animal model histological analysis of TiMg composite material for dental implants
}

\author{
Ćatić Amir, UNIZG, School of Dental Medicine, Zagreb, Croatia \\ Muhić Asja, Private dental practice, Sarajevo, Bosnia and Herzegovina \\ Beširović Hajrudin, UNISA, Veterinary faculty, Sarajevo, Bosnia and Herzegovina \\ Balog Martin and Krizik Peter, IMMM, Slovak Academy of Sciences, Bratislava, Slovakia, \\ Schauperl Zdravko, UNIZG, Faculty of Engineering and Naval Architecture, Zagreb, Croatia
}

Background: One of the main issues concerning dental implant materials arises from their Young's modulus being considerably higher than that of bone, which can generate stress-shielding effect and periimplant bone atrophy during loading. By use of advanced powder metallurgy novel titanium-magnesium (Ti12Mg) composite has been designed and consolidated. Results of ISO-standard based tests for dental implants demonstrated superior mechanical properties, in-vitro tests showed adequate biocorrosion.

Aim: The aim of the study was to produce common dimension implants and assess the invivo osseointegration potentials of Ti12Mg composite metal on animal model.

Materials and methods: Study design was presented to ethical committees of both UNIZG School of Dental Medicine and UNISA Faculty of Veterinary Medicine, and the approvals acquired. Consolidated Ti12Mg implant material was roll-pressed into a $3,5 \mathrm{~mm}$ diameter bar and machined into 12 implants of $11 \mathrm{~mm}$ length. Implant neck geometry was modeled to allow for screw-motion placement. Four sheep of various ages were chosen from natural pasture farm with HACCP certificate and kept 30 days in controlled conditions with identical pasture prior to surgery. At the day of surgery three implants were epicrestally placed using regular implant surgical protocol in right tibia of each sheep $2 \mathrm{~cm}$ apart. After 4, 8 and 12 weeks from each sheep one implant and the surrounding bone were removed by a $7 \mathrm{~mm}$ diameter trephine bur, the samples prepared and histologically analyzed. All surgeries were performed in general anesthesia. After completion of the investigation sheep were healthy and in normal function.

Results: Histological analyzes demonstrated formation of bone at the bone to implant contact. Time-related bone maturation was typical for dental implants. However, BIC at 12 weeks was more mature at Ti12Mg implants in comparison to the commercially available implant. Histomorphometric analysis showed average BIC values within the bone marrow at 4 weeks $52 \%$, at 8 weeks $81 \%$ and at 12 weeks $96 \%$. BIC values within the cortical bone were $59 \%$ at 4 weeks, $89 \%$ at 8 weeks and $98 \%$ at 12 weeks. No signs of gaseous enclosures were determined in the surrounding bone.

Conclusions and clinical implications: Proposed Ti12Mg composite, with Mg phase acting as beneficiary modulator for generating an osseoconductive surface via spontaneous dilution in body environment as well as bone formation stimulant, suggests this material answers crucial demands for dental implants. Information concerning similar studies is scarce, and implant material where porous bioactive bimetallic composite is obtained with similar properties, has not so far been proposed.
Histological analyzes demonstrated formation of bone at the bone to implant contact. Time-related bone maturation was typical for dental implants. However, BIC at 12 weeks was more mature at Ti12Mg implants in comparison to the commercially available implant. Histomorphometric analysis showed average BIC values within the bone marrow at 4 weeks $52 \%$, at 8 weeks $81 \%$ and at 12 weeks $96 \%$. BIC values within the cortical bone were $59 \%$ at 4 weeks, $89 \%$ at 8 weeks and $98 \%$ at 12 weeks. No signs of gaseous enclosures were determined in the surrounding bone.
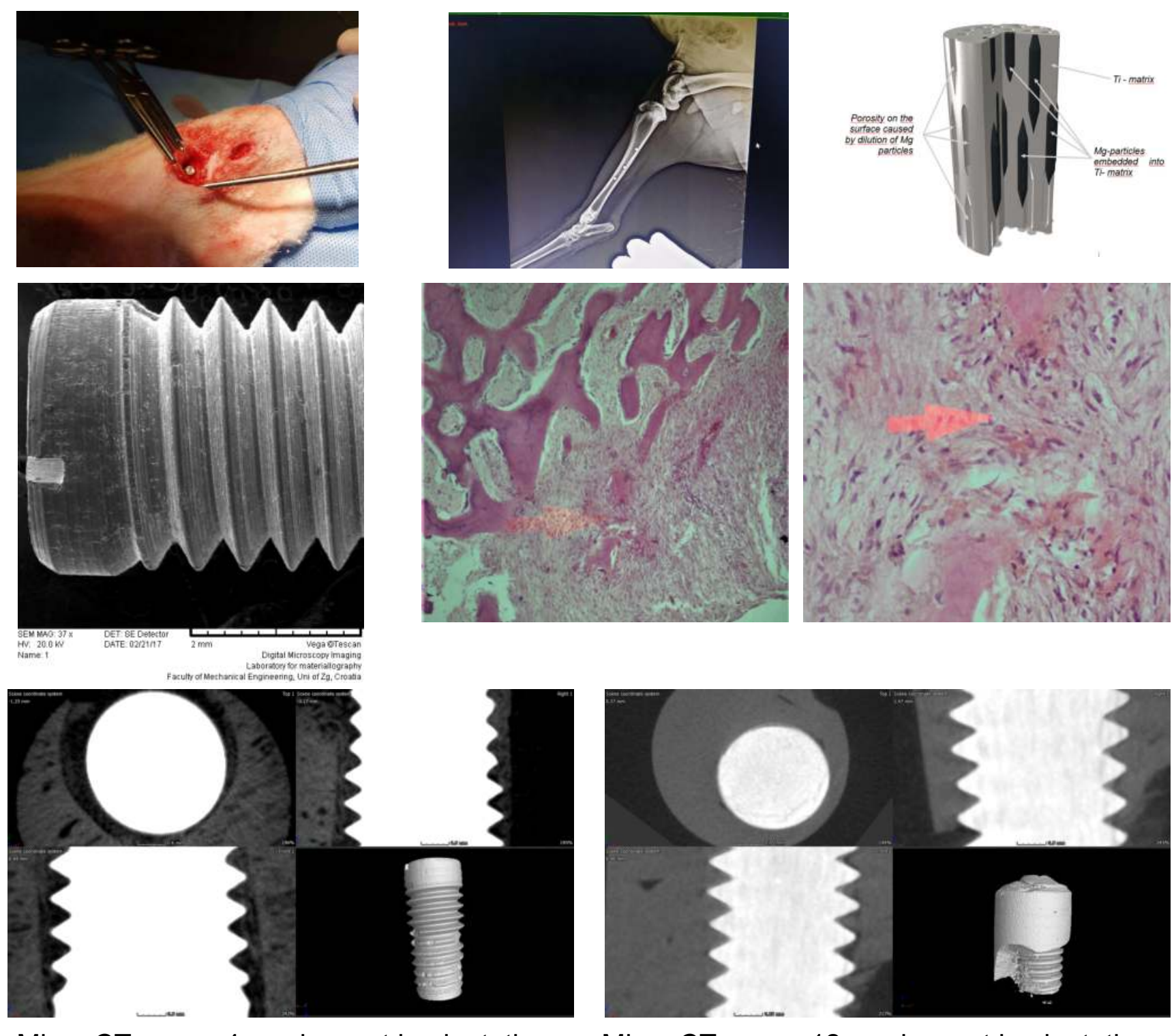

Micro CT scan - 12 weeks post implantation

\section{Background and Aim}

One of the main issues concerning dental implant materials arises from their Young's modulus being considerably higher than that of bone, which can generate stress-shielding effect and periimplant bone atrophy during loading. By use of advanced powder metallurgy novel titanium-magnesium (Ti12Mg) composite has been designed and consolidated. Results of ISO-standard based tests for dental implants demonstrated superior mechanical properties, in-vitro tests showed adequate biocorrosion.

The aim of the study was to produce common dimension implants and assess the in-vivo osseointegration potentials of Ti12Mg composite metal on animal model.

\section{Conclusion}

Proposed Ti12Mg composite, with $\mathrm{Mg}$ phase acting as beneficiary modulator for generating an osseoconductive surface via spontaneous dilution in body environment as well as bone formation stimulant, suggests this material answers crucial demands for dental implants. Information concerning similar studies is scarce, and implant material where porous bioactive bimetallic composite is obtained with similar properties, has not so far been proposed.

\section{Methods and Materials}

\section{References}

Study design was presented to ethical committees of both UNIZG School of Dental Medicine and UNISA Faculty of Veterinary Medicine, and the approvals acquired. Consolidated Ti12Mg implant material was roll-pressed into a $3,5 \mathrm{~mm}$ diameter bar and machined into 12 implants of $11 \mathrm{~mm}$ length. Implant neck geometry was modeled to allow for screw-motion placement. Four sheep of various ages were chosen from natural pasture farm with HACCP certificate and kept 30 days in controlled conditions with identical pasture prior to surgery. At the day of surgery three implants were epicrestally placed using regular implant surgical protocol in right tibia of each sheep $2 \mathrm{~cm}$ apart. After 4, 8 and 12 weeks from each sheep one implant and the surrounding bone were removed by a $7 \mathrm{~mm}$ diameter trephine bur, the samples prepared and analyzed.
Stanec, Z, Halambek J, Maldini K, Balog M, Krizik P, Schauperl Z, Ćatić, A. Titanium ions release from an

innovative titanium-agnesium composite: and in vitro study. Acta Stomatol Croat 2016; 50(1):40-48 Mine Y, Makihira S, Nikawa H, Murata H, Hosokawa R, Hiyama A, et al. Impact of titanium ions on osteoblast-,

osteoclast- and gingival epithelial-like cells. J Prosthodont Res. 2010 Jan;54(1):1-6. Wachi $\mathrm{T}$, Shuto $\mathrm{T}$, Shinohara $\mathrm{Y}$, Matono $\mathrm{Y}$, Makihira S. Release of titanium ions from an implant surface and their effect on cytokineproduction related to alveolar bone resorption. Toxicology. 2015 Jan 2;327:1-9. Balog M, Hassan Ibrahim AM, Krizik P, Bajana O, Klimova A, Ćatić A, Schauperl Z. Bioactive Ti+Mg composites fabricated by powder metallurgy: The relation between the microstructure and mechanical properties. Journal Mechanical Bech Biomed Materials 2019; 90:45-53 